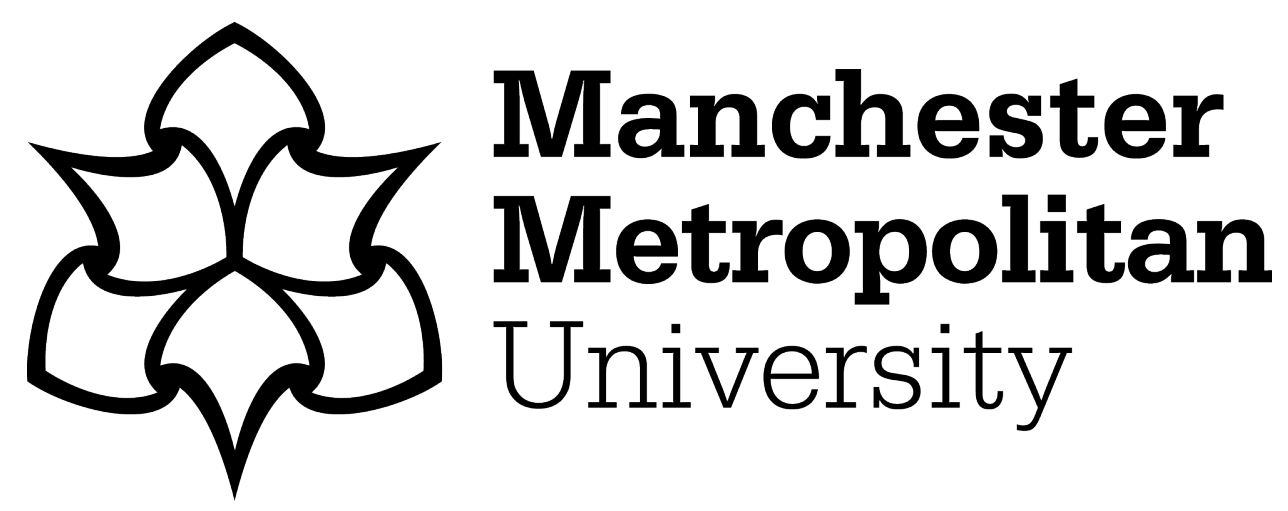

Bashir, Ali Kashif, Arul, Rajakumar, Basheer, Shakila, Raja, Gunasekaran, Jayaraman, Ramkumar and Qureshi, Nawab Muhammad Faseeh (2019) An optimal multitier resource allocation of cloud RAN in $5 \mathrm{G}$ using machine learning. Transactions on Emerging Telecommunications Technologies, 30 (8). e3627. ISSN 2161-3915

Downloaded from: https://e-space.mmu.ac.uk/622940/

Version: Accepted Version

Publisher: Wiley

DOI: https://doi.org/10.1002/ett.3627

Please cite the published version 


\title{
An Optimal Multi-tier Resource Allocation of Cloud RAN in 5G using Machine Learning
}

\author{
Ali Kashif Bashir ${ }^{*}$, Rajakumar Arul ${ }^{2 *}$, Shakila Basheer, Gunasekaran Raja, \\ Ramkumar Jayaraman, Nawab Muhammad Faseeh Qureshi
}

\begin{abstract}
The networks are evolving drastically since last few years in order to meet user requirements. For example, the $5 \mathrm{G}$ is offering most of the available spectrum under one umbrella. In this work, we will address the resource allocation problem in 5G networks, to be exact in the Cloud Radio Access Networks (C-RAN). The Radio Access Network (RAN) mechanisms involve multiple network topologies that are isolated based on the spectrum bands and it should be enhanced with numerous access technology in the deployment of 5G network. C-RAN is one of the optimal technique to combine all the available spectral bands. However, existing C-RAN mechanisms lacks the intelligence perspective on choosing the spectral bands. Thus, C-RAN mechanism requires an advanced tool to identify network topology to allocate the network resources for substantial traffic volumes. Therefore, there is a need to propose a framework that handles spectral resources based on user requirements and network behavior. In this work, we introduced a new C-RAN architecture modified as multi-tier H-CRAN in a $5 \mathrm{G}$ environment. This architecture handles spectral resources efficiently. Based on the simulation analysis, the proposed multi-tier H-CRAN architecture with improved CU in network management perspective enables augmented granularity, end-to-end optimization, and guaranteed Quality of Service (QoS) by 15 percentages over the existing system.
\end{abstract}

Keywords - Radio access network, Quality of Service, Baseband Unit, Quality of experience.

\section{Introduction}

In the midst of this data revolution era, the evolutions in the network generations should be steered largely to hold up faster data-oriented services that can be fulfilled in 5G. The forthcoming network is planned to evolve based on the user expectation and demands which in turn forms the basement for the 5G network goals. Some of the predominant targets of 5G are low latency, high bandwidth utilization and interoperability. To achieve the significant goals of 5G, efficient network management and compatible network architecture is required. The $5 \mathrm{G}$ network incorporates various emerging technologies like Software Defined Networking (SDN), Network Function Virtualization (NFV) and Mobile Edge Computing (MEC) [1] which were proposed by the main working group of $5 \mathrm{G}$ networks like NGMN and $3 \mathrm{GPP}$. The $5 \mathrm{G}$ consortium has planned to utilize the available spectrum of all the communication and data sharing bands of the licensed spectrum such as LTE, UMTS and unlicensed spectrum like WiFi [2]. The advanced antennas with Multiple Input and Multiple Output (MIMO) technologies can be adopted in the evolving 5G to provide higher data rate [3] and [4]. The colossal requirements of future $5 \mathrm{G}$ networks cannot be accomplished by the existing Radio Access Networks (RANs), wherein the Base Band Units (BBUs) and Radio Units (RUs) are consolidated [2].

Meanwhile, the cloud-enabled services in 5G ecosystem cater heterogeneous communication framework utilizing advanced virtualization techniques. The network virtualization stimulates multiservice and multi-tenancy for efficient network operations and service provisions which in turn offers new-fangled service-oriented and edge-cloud 5G architectures with enhanced Quality of Experience (QoE) [5]. Thus, the current RAN architectures need to be further extended with various virtualization techniques to develop Cloud RAN (C-RAN). C-RANs abutments the correlation of Access Points (APs) to the pool of BBU via a troop of transport links in the Control Unit (CU) [6] which conquers the 
limitations in the traditional RAN [7]. However, C-RAN requires an intelligent technique to recognize the topology of the network in the mobility stage and to locate the resources, as the vast traffic volume created by the sampled radio signals are directly transported to the CU [8].

The next-generation networks are expected to learn consistently based on the varied user behavior and spectral stability that depends on both the user's environment and network behavior [9]. Thereby seamless service can be ensured for the needy as per the requirement by predicting the most advantageous network that can render service [10]. The chosen optimal network will offer seamless service, with highly sophisticated learning and decision-making through machine learning techniques. Machine learning is the most robust artificial intelligence domain which comprises of assured techniques for improving the operation and management of networks and engineering applications in the future cellular networks. The machine learning algorithms employed for mobile networks can endeavor plentiful opportunities to solve advanced threats and enhance connectivity [11]. Hence, the machine learning aided C-RAN architectures are expected to perform as an intelligent radio propagation medium which can automatically assess the available spectrum and can regulate the power to be transmitted in the interest of energy conservation. It is believed that an innovative C-RAN with machine learning technique forms a new design prototype in the $5 \mathrm{G}$ network architecture which will most probably introduce considerable modifications in the air interface and the tensile network management. Thus, CRAN invites a fruitful solution that solves the issue of interoperability by utilizing various spectrum bands by learning the dynamic changes in the networking paradigm. In this article, we proposed a Multi-tier $\mathrm{H}$-CRAN in $5 \mathrm{G}$ by adopting the machine learning techniques to address the current limitations of huge traffic in the BBU pool of existing architecture. The proposed architecture is also improved to facilitate augmented granularity, end-to-end optimization, and guaranteed Quality of Service (QoS). This article is organized as, section 2 discusses the literature on the recent research solution that contributes to the domain of $5 \mathrm{G}$ and machine learning. The feasibility in the physical Deployment of RAN in heterogeneous networks is explained in section 3. In section 4, proposed Multitier H-CRAN architecture with learning tier is briefed. The performance of the proposed system is analyzed in section 5 under various assumptions and 5G scenarios. Finally, section 6 concludes the article with the inference of analysis made on the proposed system.

\section{Related Works}

The $5 \mathrm{G}$ wireless network is evolving to satisfy the end user requirements such as seamless connectivity even with high mobility, low latency and interoperable connectivity. The mobile communication working groups and consortiums focus on providing new spectrum bands, on improving spectral efficiency and throughput when considering the business models related to coverage area and reliable broadband access [12]. To meet the user requirements, RAN is redesigned to scale the parameters such as throughput, many devices and connections concerning User Plane (UP) and Control Plane (CP). The evolving 5G RAN architecture supports mechanism that relates traffic, to fulfill Quality of Service (QoS) and to control D2D connectivity [13]. RAN technology also assists in reducing traffic services and enables network slicing [14]. However, there is a possibility for RAN to be designed up with heterogeneous networks like WiFi and LTE small cells, i.e. HetNets [15].

By the recent advancements in the cloud computing paradigm, the integration of Cloud technology with RAN named as C-RAN is believed to be a productive solution to solve the heterogenous interoperable problems. C-RAN is the most promising design for the mobile network system to provide scalable and smooth traffic transmission. C-RAN integrated with HetNets has achieved flexible and reconfigurable dense deployment of small cells. C-RAN holds the potential in giving effective communication and 
information exchange among the terminals. When C-RAN is modeled with the assistance of SDN, it gives a space to configure and manage the dense small cells deployment [16] and [17]. To enhance the QoS in C-RAN, BBU pool is relocated into BBU and Remote Radio Head (RRH) where BBU is enabled with the central processing unit to provide network coordination and management. BBU helps in reducing the traffic when deploying the small cells, i.e. HetNets which contains thousands of small cells. In BBU, there is a possibility of several other challenges like effective radio resource management, spectrum, and energy efficient network to enable QoE, resource management in small cells and multi-hop networks.

By considering cooperative radio resource management, a modified C-RAN framework has been proposed to provide an improved indulgent of resource allocation. It also enables flexible resource management with improved QoS parameters such as throughput and fairness [18]. The modified C-RAN framework allows flexible resource management, i.e. the amount of resource used for the end users is made dynamic. Several issues came into existence when C-RAN is combined with HetNets such as coordinated multipoint transmission and reception, resource management in self-organized networks and improved spectrum utilization with energy estimation in cellular systems. In C-RAN, the transmission demand between BBU and RRH are considered for the differentiation of services and RRH demand in the transmission links, i.e. backhaul is improved by balancing the workload which is labeled as multipoint transmission [19]. In C-RAN, BBU and Radio Access Units (RAUs) are isolated to provide centralized processing that reduces operational expenses. While mapping RRH and RAUs in heterogeneous networks, the hardness of configuration and energy efficiency are the prime parameters to be considered [20]. C-RAN along with Orthogonal Frequency Division Multiple Access (OFDMA) technique, helps to map BBU and RAU for providing reconfigurable backhaul transmission and effective energy utilization. Efficient pre-coding scheme [21] has been designed for the environment like C-RAN with full channel matrix using beamforming i.e. zero-forcing to reduce energy consumption and computational complexity in cooperative transmission. Centralized processing of thousands of small cells helps to perform multiple cell joint scheduling, but interference problem and frequency reuse play a significant role in the dense scenarios [22].

Next generation networks are often planned for the improved data rate which is used for many essential applications like sensing, resource management, etc. Challenges faced by radio technology has to provide creative learning and decision making platform for efficient frequency planning. Based on radio frequency spectrum, a heterogeneous network meets spectral inefficiency, interference transmission and power inefficiency concerning QoS [23] and [24]. HetNets faces the problem of inefficient radio resource management and optimization for the user's satisfaction [25]. To overcome the various network degradation problems, artificial intelligence techniques such as machine learning, fuzzy neural networks, etc. play an important role to provide practical solutions [26]. When artificial intelligence is deployed on the C-RAN by integrating HetNets, Base Stations (BS) uses dedicated backhaul to provide guaranteed backhaul and small cells deployment offers seamless connectivity to offload the data in traffic. If C-RAN is automated or made intelligent, then RRH in backhaul connection of RAN can be combined with the BBU pool to create a centralized processing unit [27].

Due to the tremendous traffic generated by mobile users, the future mobile network deployment is going to be extremely complex. The spectrum utilization in the densely populated network is a challenging task and if machine learning [28] is used as an optimization tool to optimize the C-RAN, the resource allocation scheme will be enabled to downlink H-CRAN [29]. H-CRAN aims the reduction of an interference problem to maximize the energy efficiency and to guarantee the QoS between RRH and all UE's present in the network. In this paper, we propose a multi-tier H-CRAN architecture, that can provide seamless connectivity by monitoring the network conditions and user behavior based on the 
behavioral pattern, to avail the required technology in the future. Also, the proposed multi-tier H-CRAN considers the problem of interference to get optimized resource allocation further to improve the QoS.

\section{Deployment of RAN in HetNets}

The key idea of our proposal is to deploy RAN in the scenario of HetNets. In order to buckle with the scope of small cells prosperity, Cloud Radio Access Networks (C-RANs) with minimized Captial Expenditure (CAPEX) and Operating Expenditure (OPEX) is proposed. C-RANs have independent Baseband Units (BBUs) and independent RAUs. These standalone units are migrated from centralized processing and are placed in the cloud. The backhaul that connects the BBU and RAU is said to be the principal component of C-RAN. For efficient network management, the 5G leverages SDN, NFV and Mobile Edge Computing (MEC) principles. The large requirement of future 5G networks cannot be accomplished by the existing Radio Access Networks (RANs), wherein the baseband units (BBUs) and radio units (RUs) are consolidated. Cloud RANs abutments the correlation of Access Points (APs) to the pool of BBU via a troop of transport links in the Control Unit (CU) which conquer the limitations in the traditional RAN [6]. However, C-RAN requires an intelligent technique to recognize the topology of the network in the mobility stage and to locate the resources, as the vast traffic volume created by the sampled radio signals are directly transported to the CU. Initially, machine communication services are categorized as massive Machine-Type Communications (mMTC), enhanced Mobile BroadBand (eMBB) and ultra-reliable, Low-Latency Communications (UR-LLC) services [30] which have very different performance requirements and traffic profiles. Thus the litheness and extensibility introduced by this new C-RAN architecture grants to deviate the vision from the generic C-RAN to Multi-layer C-RAN. In the multi-layer C-RAN, UE assignments should be dealt with adequate dormancy to solve the UE latency and energy utilization problems in RRHs.

Thus, we propose a novel architecture involving multi-layer of C-RAN network as multi-tier H-CRAN. The proposed architecture is also equipped with unique control and innovative management schemes to facilitate augmented granularity, end-to-end optimization, and guaranteed Quality of Service (QoS). To support these new use cases, in this work, we inherent machine learning paradigm with CRAN deployment for high scalability and flexibility. So, the next generation architecture extends the cloud RAN technology to ultra-dense networks that incorporate macro, micro and small cells in diverse spectrum bands, i.e. HetNets.

\subsection{Generalization of HetNets:}

HetNets incorporates a bulk quantity of compactly deployed small cells such as femtocells, picocells, Relay Nodes (RN), LTE and WiFi Access Points (APs) [31] and [32]. These small cells can serve both as indoor and outdoor UEs. These are deployed in the densely colonized region to reconcile the immense traffic cravings. Picocells maneuver on licensed bands and use either optical fiber or microwave interface as backhaul to get connected with the core network. They are set up by the cellular speculator, either in an organized or in an unorganized way, at numerous traffic hot-spots. Femtocells are habitually functioning on licensed bands and exploit the broadband Internet linkage as backhaul. The core network is then linked employing either extant Digital Subscriber Line (DSL) or cable modem done with the IP network. They are incorporated by human beings predominantly on the way to deliver interior coverage. Relay Node (RN) is the stretched version of its benefactor evolved Node B (eNB), it is linked with the benefactor eNB through the mediator $U_{u}$ amalgamator in addition to the radio link. Backhaul is capable of exploits either clonal frequency band (Inband) as being pre-owned in air 
mediator or is capable of exploiting peculiar band (Outband). UE is coupled with $\mathrm{RN}$ through the Uu mediator. When a UE does not become familiar with the case it is associated to relay or network then the relay will be translucent, or else when the UE is familiar with the case it is associated to network or relay, the relay will be non-translucent. Wi-Fi APs are small cells habitually functioning on unlicensed ISM bands with the frequency between $2.4 \mathrm{GHz}$ and $5 \mathrm{GHz}$. WiFi APs are incorporated either by a manipulator or human beings to afford a cramped coverage zone and can be positioned indiscriminately in the interior of every cell, which has the capacity of transmitting power varies from $100 \mathrm{~mW}$ to $200 \mathrm{~mW}$. It makes use of the broadband Internet connection as backhaul. LTE [5] small cells are endorsed because of organization litheness, curtailed capital, and operational costs, in addition to the diminished energy regime. [6] As a result of their supplementary compressed arrangement feature, the LTE network is alienated into two parts: the LTE part handles radio access expertise, while the Evolved Packet Core (EPC) handles the expertise allied to a core network. A UE fixes to an eNodeB by means of a radio mediator. The eNodeB executes the administration of radio resource tasks such as allotting radio resources in addition to the administration of inter-cell interference.

The general network architecture differentiating HetNet, C-RAN and H-CRAN is depicted in Fig. 1. By scheduling transmissions centrally, C-RAN can improve cell edge performance by the addition of reducing the inter-sit interference and can serve the users across different radio access bearers. With inter-site connectivity and de-coupled uplink and downlink, cloud RAN introduces the concept of the "no-edge" network to ensure consistent improved user experience in high-density networks. The central controller of the C-RAN can be designed using "cloud-native" software to run-on general-purpose telco cloud infrastructure. The placement of the controller corresponds to an edge data centre

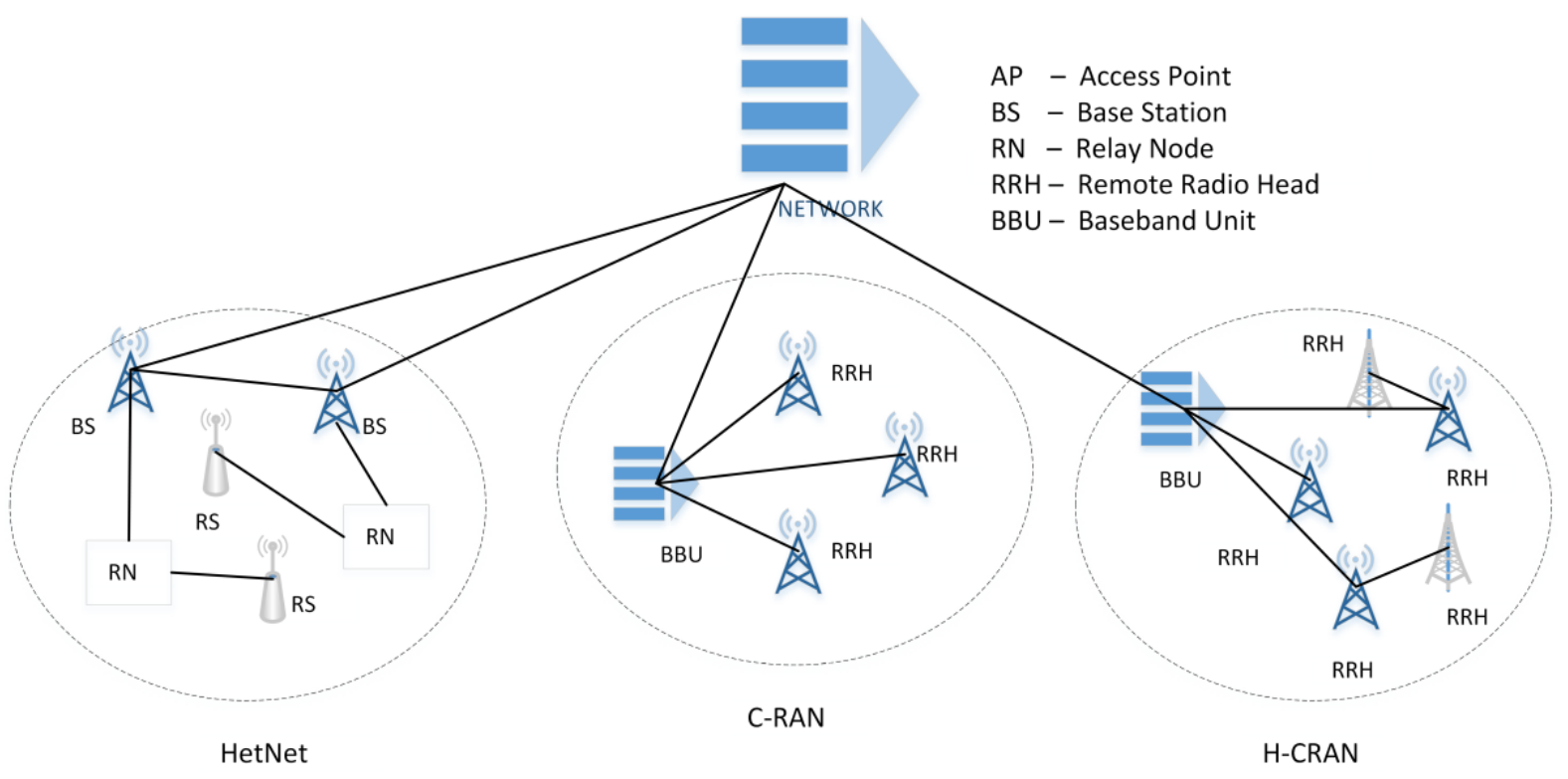

Fig. 1. Network Architecture

facility that is capable of running Multi-access Edge Computing (MEC) services. One opportunity is to consider how radio access and cloud based MEC services can be integrated. For instance, to enable developers and content providers to optimize their service, according to the desired or available radio bandwidth. It is expected to be useful for ultra-low-latency and mission-critical services in $5 \mathrm{G}$ and it is being formalized with the $\mathrm{CU}$ and DU functional split. On the DU side, for example, the use of massive MIMO associated with beam-tracking and beam-switching techniques require highly accurate time 
estimation to support mobility, which points toward distributed real-time radio function as optimal. Artificial intelligence techniques are believed to be an optimization tool to optimize the C-RAN in the network.

\subsection{Automated, Intelligent OA\&M:}

A critical capability of the next-generation cloud-RAN architecture is the ability to accurately understand the network status in real time across the coverage area [33]. Operations, Administration and Management (OA and $\mathrm{M}$ ) automation is vital because of the significant operational cost dedicated to ongoing RAN optimization. Coordinated scheduling can radically improve deployment flexibility and performance. It also enables operators to:

- Automate continuous radio optimization

- Accelerate network expansion and deployment

- $\quad$ Adapt to changing user behavior

By integrating cloud based network in RAN the control functions in the network are analyzed to quicken network expansion and implementation, thus in overall, it improves QoS and QoE.

\subsection{Edge Cloud Deployment \& Integration:}

Cloud RAN offers an opportunity to integrate radio access with the rest of the telco cloudenabled network -for example, using ME [34]. In this model, the application logic or content is hosted on cloud infrastructure at the same edge data centre facility as the centralized control functions. It is particularly useful where an application is delay-sensitive. When the service is not time-critical, it may be more efficient to process data centrally. For the commercial network deployment, dual connectivity plays a vital role by introducing LTE and 5G RAN network.

\subsection{G NR with Dual-Connectivity to LTE:}

A non-standalone mode is a way to add 5G New Radio into an LTE network [35] and [36]. The LTE RAN is used as a Master evolved NodeB (MeNB) and provides control in signalling to establish and manage the connection with the devices. It is likely to be the way $5 \mathrm{G}$ is introduced into commercial networks by the network operators. By analysing the above terminologies and technologies, we found the challenges as follows,

1. In Multi-layer CRAN, UE assignment should be dealt with adequate dormancy.

2. In H-CRANs, there are many challenges ahead, including optimal resource allocation over the constrained front haul, energy harvesting, backward compatible standards development, and so on.

3. To our best knowledge, no existing work has investigated the benefits of cross-tier and intra-tier cooperation under the capacity limitation in a data frame. The challenges come from how to utilize radio resource efficiently and to enhance cooperative gain conforming to the constraints of capacity in H-CRAN

4. The frequency resources of H-CRAN is distributed among the RRHs and HPNs which makes the optimization to serious inter-tier interference issues and non-convexity issue. 
5. In complicated heterogeneous networks as we see in the 5G deployment, where many RRHs are multi-hop connected to the BBU pool, a significant problem that must be solved is front haul resource management, especially for front haul resources shared by hotspot RRHs.

6. The concern now is how operators can be best to make use of virtualization technologies in RANs while at the same time building cost, spectrum and energy efficient networks that offer a seamless user experience.

Based on these pieces of literature in section 3, we have reconstructed the existing C-RAN architecture to figurate the new proposed structure based on the integration of multi-tier component such as physical, cluster and machine learning strategies as in Fig. 2.
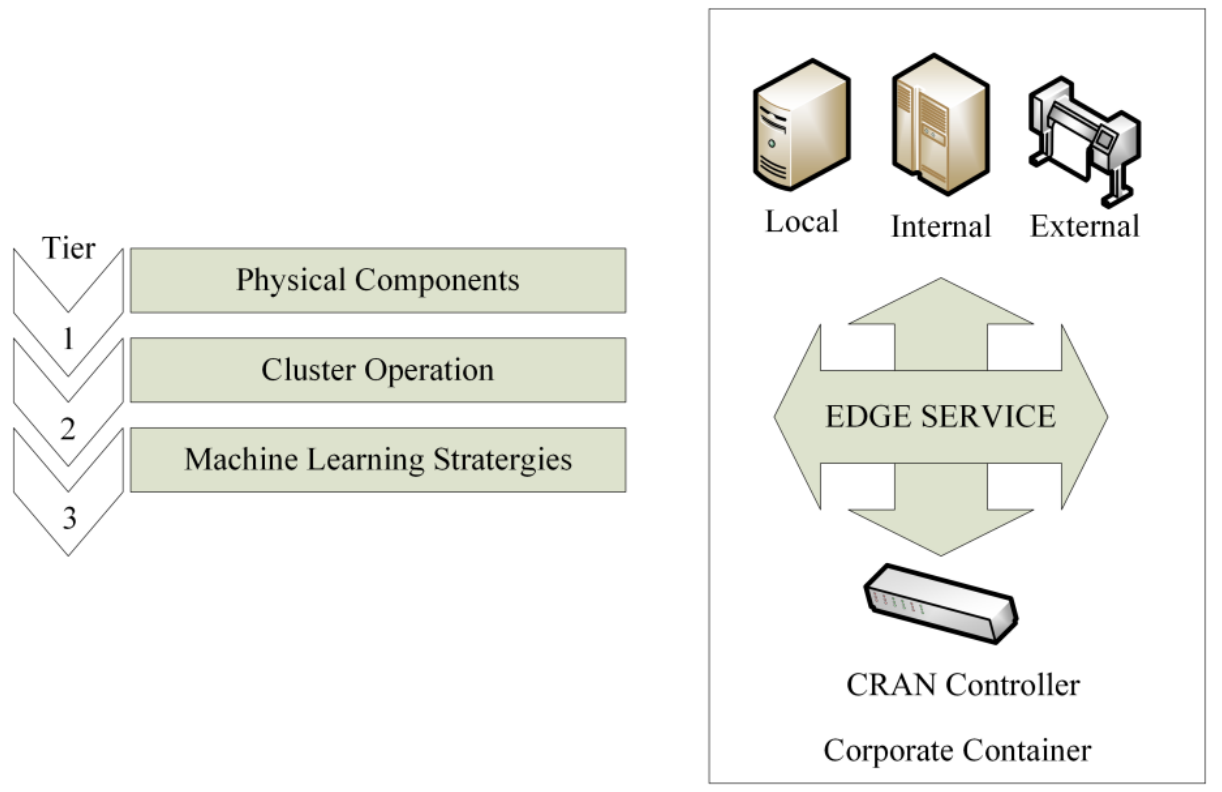

Fig. 2. Proposed Vs Actual network system

For the integration of machine learning with C-RAN, HetNets such as picocells and small cells, etc. requires a self-configured / optimized network. To build such a framework, we need to solve the problem of resource allocation and interference coordination problem in either uplink/downlink networks. In this paper, we have addressed the resource allocation problems that exist when CRAN is operated with heterogeneous networks through machine learning strategies. In this paper, we have applied Q-Value approximation algorithm, a learning strategy is integrated with resource allocation process to provide effective decision based on the calculation on QoS metric. Also, the selection of active RRH cell to provide allocation of resource in the network is represented in algorithm 2 follows a modelled graph coloring methodology. The challenges that will be faced in the considered scenario is diagrammatically represented in Fig. 3. 


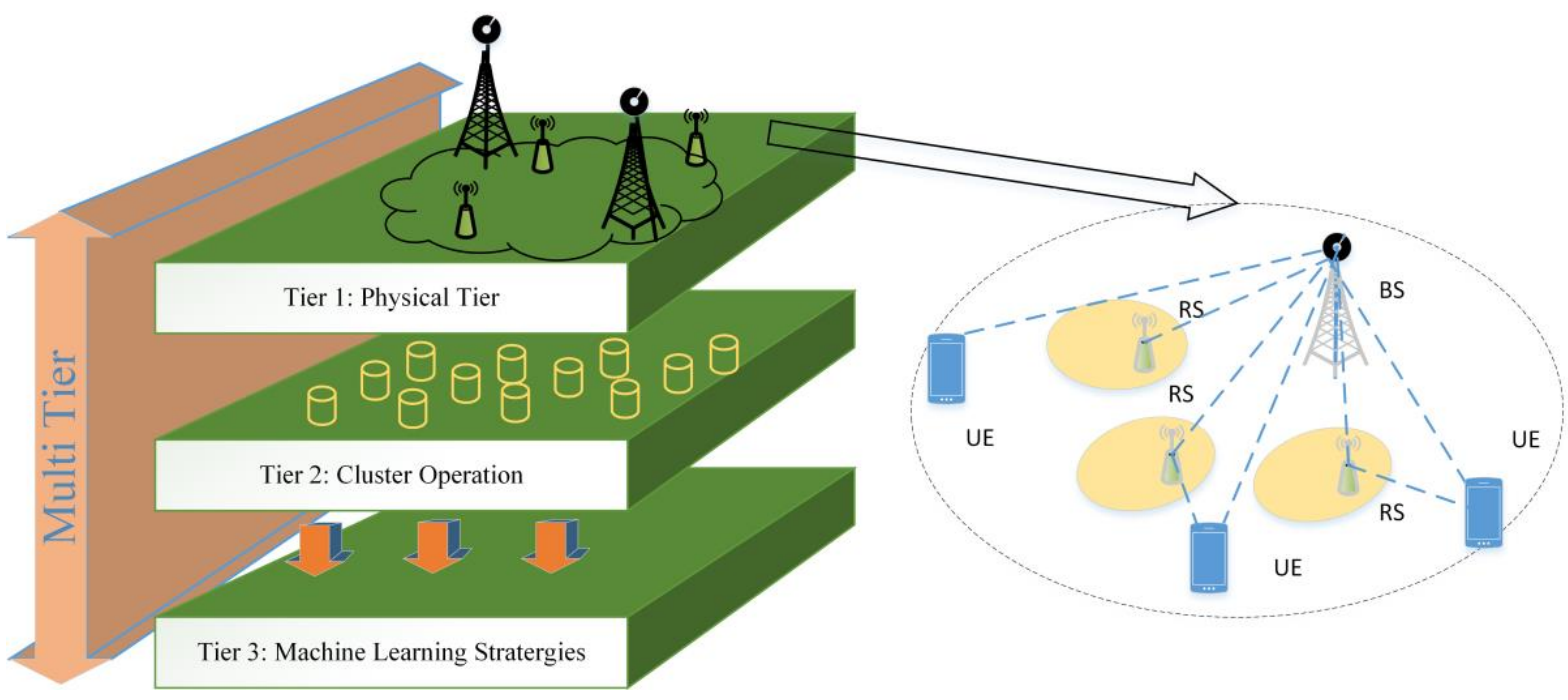

Fig. 3. Multi-Tier C-RAN HetNets Structure

\section{Co-ordinated UE Multi-tier C-RAN}

In this article, we propose Co-ordinated UE Multi-tier H-CRAN, that is deployed in the CRAN technology for the selection of desirable network based on user behavior which is represented as Algorithm 1. The Algorithm 1 takes the required bandwidth and data rate as the input to provide an accurate selection of network technology. In fig. 4, the UE assignments should be dealt with Multilayer H-CRAN with adequate dormancy. The total number of UEs is to be increased in the optimization issue of RRH cell, for the sufficient dormancy in each cell. The Co-ordinated UE algorithm, supportively communicates and computes the resource allocation task of each RRH cell which leads to minimized UE assignment dormancy and decreased the cost of energy. A distributed way of cell colouring based resource allotment methodology is followed by RA to facilitate all RRH cell for distributed resource allotments which is completed through algorithm 2. In algorithm 2, one colour is assigned to 1 active cell and repeated to all the cells in the set of cells. In the next repetition, it is moved to the next colour in the set of cells to compute the utility of the network and QoS metric. Algorithm 2 helps to assign colour next to next until no resource allocation in the network is possible. Along with the resource allocation, we have adapted learning strategy, i.e. approximation Q-value learning algorithm [26] to provide resource allocation effectively to improve QoS to the users in the network. Finally, the resource allocation of every RRH cell is computed. In this Co-ordinated UE system, we have discussed two cases such as interference and non-interference coordination. 


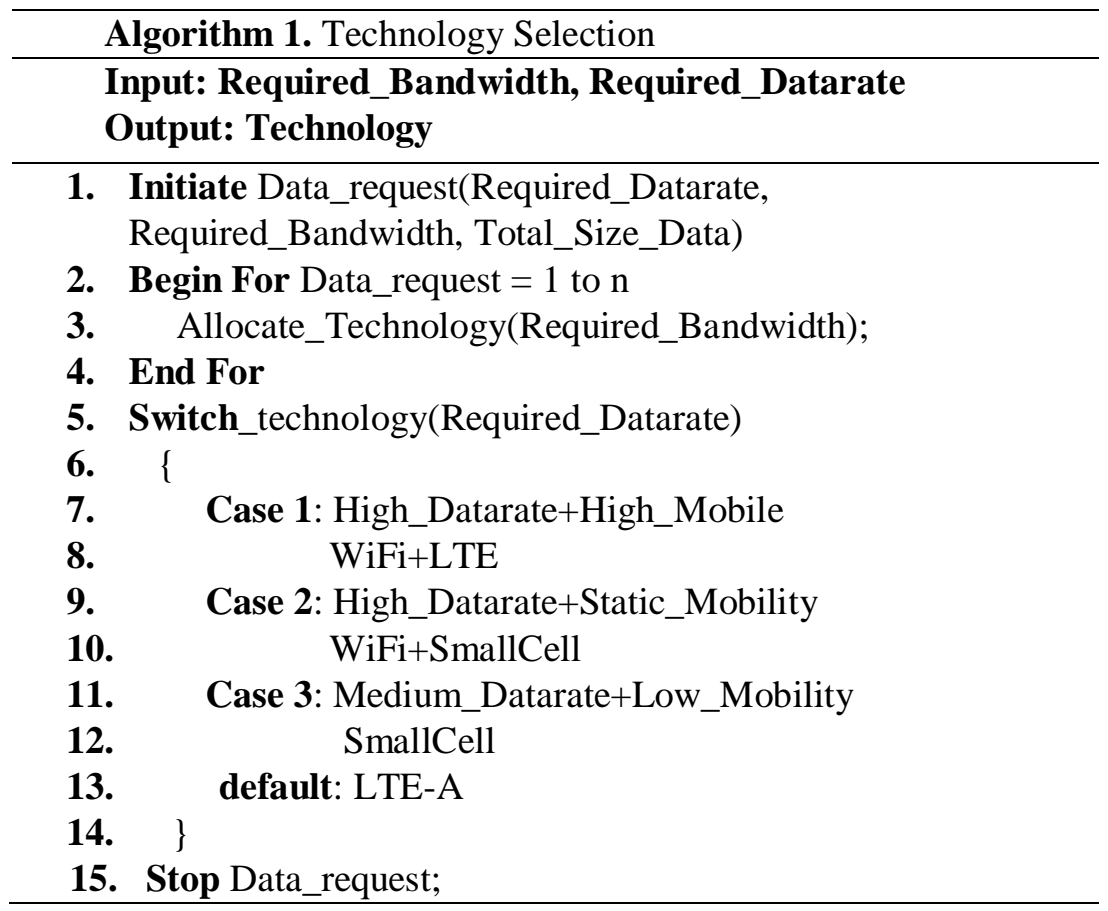

In the BBU pool of H-CRAN, the frequency resources are distributed among many RRHs and a single HPN. For a ranted segment of frequency resources and time slot, the RRH rosters only a single UE. Consider the following Gaussian Interference Channel, in which let the power transmission of HPN is said to be $\mathrm{PH}$. Node A and B are communicating with each other to access different frequency resources $\mathrm{F}$ to $\mathrm{C}$ and $\mathrm{D}$ at the edge of the cell by enabling the cell-edge concept in our proposed work to avoid interference. A and $\mathrm{B}$ will receive information by the respective transmitter $\mathrm{C}$ and $\mathrm{D}$ nodes.

$\mathrm{F}=\mathrm{f}_{1}, \mathrm{f}_{2}, \ldots . \mathrm{F}_{3}$

For the UE of $i^{\text {th }} \mathrm{RRH}$ and $u^{\text {th }} \mathrm{RRH}$,

$\operatorname{SINR}_{i, u}=\frac{t p_{i} c g_{i i}}{\sum_{h \neq i} t p_{h} c g_{i h}+T P_{H P N} c g_{i}^{H P N}+d_{i}^{2}}+\frac{t p_{u} c g_{u u}}{\sum_{v \neq u} t p_{v} c g_{u v}+T P_{H P N} c g_{u}^{H P N}+d_{u}^{2}}$

Where $t p_{i}$ represents the power transmission of the $i^{\text {th }} \mathrm{RRH}, c g_{i h}$ represents the hike of the carrier among the $i^{\text {th }} \mathrm{RRH}$ and the UE of the $h^{\text {th }} \mathrm{RRH}$. $T P_{H P N} c g_{i}^{H P N}$ and $T P_{H P N} c g_{u}^{H P N}$ stands for the inter-tier interference where $T P_{H P N}$ represents the power transmission of the HPN and $c g_{i}^{H P N}$ in (1). Also $c g_{u}^{H P N}$ stands for instruction about the state of the carrier among the UE of the $t^{\text {th }}$ and $u^{\text {th }} \mathrm{RRH}$ and the HPN. $d_{i}^{2}$ and $d_{u}^{2}$ is the noise power spectral density.

When a transmission selection is applied in each BBU, transmission serves from $\mathrm{i}^{\text {th }} \mathrm{RRH}$ to $\mathrm{u}^{\text {th }} \mathrm{RRH}$ at a particular time ' $t$ '. SINR computation helps $i^{\text {th }}$ RRH mapped to $u^{\text {th }}$ RRH of a cluster as represented in (1) as represented to minimize the interference based on SNR.

A positive matrix $W M$ and $W F$ is specified by the candidates:

$C=\beta \cdot W \cdot \log _{2}(1+\alpha \cdot S N I R)$

In (2), we have formulated channel capacity which is nothing but the throughput by considering the bandwidth (W) and data rate $(\beta)$ with a signal to noise ratio (SNIR) with the constant $(\alpha)$ with 0.1 to 1 after calculating the SNIR. 
$W M=\left\{\begin{aligned} 0, & i=h \\ \frac{c g_{i h}}{c g_{i i}}, & i \neq h\end{aligned}\right.$

$W F=\left\{\begin{array}{l}0, u=v \\ \frac{c g_{u v}}{c g_{u u}}, u \neq v\end{array}\right.$

Where, WM and WF are the channel vectors derived from inter-tier interference based on the state of the carrier to reduce network power. It is obtained using two cases of mapping between $\mathrm{i}^{\text {th }} \mathrm{RRH}$ among the carrier and $h^{\text {th }} R R H$ of UE and mapping between $U^{\text {th }}$ and $V^{\text {th }} R R H$ and VPN as represented in (3) \& (4).

and the vectors for $W M$ and $W F$ are represented in (5).

$a_{i}=\left(\frac{V_{1}}{c g_{11}}, \frac{V_{2}}{c g_{22}}, \ldots \ldots . \frac{V_{I}}{c g_{I I}}\right)^{T}$

\begin{tabular}{l}
\hline Algorithm 2. Resource allocation in multi-tier H-CRAN \\
\hline Input: Colouring the distributed RRH cell \\
Output: Resource allocation for each RRH cells \\
\hline
\end{tabular}

1. Initialize $\mathrm{i}=1, \mathrm{C}_{\mathrm{o}}=0, \mathrm{C}_{\mathrm{n}}=1, \mathrm{~s}=0$;

2. While $\left(\mathrm{C}_{\mathrm{o}}>\mathrm{C}_{\mathrm{n}}\right) \& \&\left(\mathrm{~s}<\mathrm{S}_{\max }\right)$

3. Begin

4. $\mathrm{C}_{\mathrm{o}}=\mathrm{C}_{\mathrm{n}}$;

5. For $(\mathrm{i}<=\{$ set of cells $\}$

6. Begin

7. Label the cells with colours and order the colours

8. Choose the active cell

9. For Each active cell has certain pattern [37]

10. Begin

11. Find the potential assignments of mobile cell to the cell constraints

12. Compute utilities [38]

13. Compute the QoS metric i.e. Blocking rate

14. Then next colour activated in the next active cell

15. End

16. QoS gets improved for each loop until threshold exceeds

17. End

18. $\mathbf{C}_{\mathrm{n}}=\sum_{\forall j \epsilon C} C_{j}$

19. Increment $i+1 \%$ Set of cells $\}$

20. Increment $s+1$

21. End

22. Return $\left(\alpha_{j}, \beta_{j}\right) \forall j$ Belongs to $C$

$a_{u}=\left(\frac{V_{1}}{c g_{11}}, \frac{V_{2}}{c g_{22}}, \ldots \ldots \cdot \frac{V_{U}}{c g_{U U}}\right)^{T}$

Where $V_{I}=T P_{H P N} c g_{i}^{H P N}+d_{i}^{2}$ and $V_{U}=T P_{H P N} c g_{u}^{H P N}+d_{u}^{2}$ The $S I N R_{i, u}$ can be transformed as in (7). 
$\operatorname{SINR}_{i, u}=\frac{t p_{i} c g_{i i}}{\sum_{h \neq i} t p_{h} c g_{i h}+V_{I}}+\frac{t p_{u} c g_{u u}}{\sum_{v \neq u} t p_{v} c g_{u v}+V_{U}}$

All the set of vectors are merged as ai at the time ' $T$ '. (6) is transformed based on the vectors in $a_{u}$ in (7).

Then, recomputing (1) based on the set of vectors obtained from (7) to calculate SNIR of $\mathrm{i}^{\text {th }}$ and $\mathrm{u}^{\text {th }}$ RRH and HPN among UE which is represented in (8).

$$
\begin{aligned}
& \operatorname{SINR}_{i, u}=\frac{t p_{i}}{\sum_{h \neq i} t p_{h} \frac{c g_{i h}}{c g_{i i}}+\frac{V_{I}}{c g_{i i}}}+\frac{t p_{u}}{\sum_{v \neq u} t p_{v} \frac{c g_{u v}}{c g_{u u}}+\frac{V_{U}}{c g_{u u}}} \\
& \operatorname{SINR}_{i, u}=\frac{t p_{i}}{t p_{i} W M+a_{i}}+\frac{t p_{u}}{t p_{u} W F+a_{u}}
\end{aligned}
$$

Where $t p_{i}=\left(t p_{1}, t p_{2}, \ldots \ldots t p_{i}\right)^{T}$ and $t p_{u}=\left(t p_{1}, t p_{2}, \ldots \ldots t p_{u}\right)^{T}$

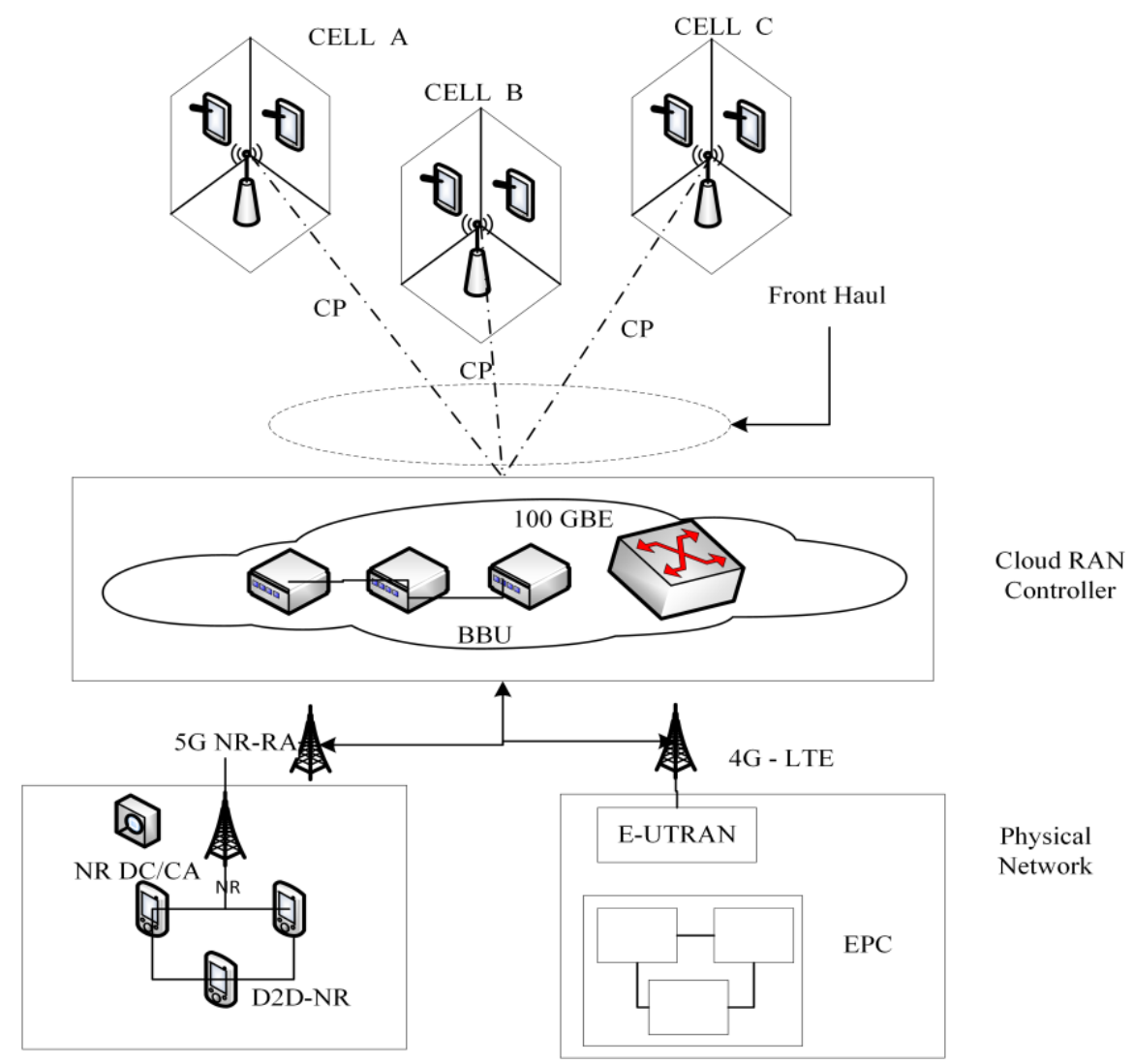

Fig. 4. Multi-Tier C-RAN Architecture

The proposed work enables cell edge concept by computing SNIR concerning the mapping between the $i^{\text {th }}$ RRH mapped to $u^{\text {th }}$ RRH of a cluster. This SNIR computation assists in reducing the interference there by considering two cases of with and without interference strategy to optimize the network performance. 


\section{A. With the strategy of Interference}

In this section, we have computed transmission power 'tp' based on RRH and HPN among the UE to optimize the power of transmission and to minimize the interference between RRH and HPN.

With the strategy of interference, the optimization issue can be dispatched in (9).

$\max _{\left\{t p_{i}, t p_{u}\right\}} \sum_{i, u=1}^{I, U}\left(w t_{i}+w t_{u}\right) \log \left(1+S I N R_{i, u}\right)$

Such that, $\sum_{i, u=1}^{I, U} t p_{i}, t p_{u} \leq T P_{\max }$

$t p_{i}, t p_{u} \geq 0$

Where $w t_{i}$ and $w t_{u}$ are the density of any UE and $T P_{\max }$ is the admitted ultimate power of transmission for the architecture and $V_{I}$ and $V_{U}$ in (9) is given as $V_{I}=d_{i}^{2}$ and $V_{U}=d_{u}^{2}$. Now, the problem can be transformed in (10):

$\max _{\left\{t p_{i}, t p_{u}\right\}} \prod_{i, u=1}^{I, U}\left[\left(\frac{\sum_{h \neq i} t p_{h} c g_{i h}+V_{I}}{\sum_{i=1}^{h} t p_{h} c g_{i h}+V_{I}}\right)^{w t_{i}}+\left(\frac{\sum_{v \neq u} t p_{v} c g_{u v}+V_{U}}{\sum_{u=1}^{v} t p_{v} c g_{u v}+V_{U}}\right)^{w t_{u}}\right]$

Such that, $\sum_{i, u=1}^{I, U} t p_{i}, t p_{u} \leq T P_{\max }$

$t p_{i}, t p_{u} \geq 0$

\section{B. The strategy without Interference}

In the strategy without interference category, it is recognized that, there is possible for interference in between HPN and RRHs. Hence it is modelled as:

$\max \sum_{i, u=1}^{I, U}\left(w t_{i}+w t_{u}\right) \log \left(1+\operatorname{SINR}_{i, u}\right)$

Such that, $\sum_{i, u=1}^{I, U} t p_{i}, t p_{u} \leq T P_{\max }$

$\sum_{i=1}^{I} c g_{i}^{A} t p_{i} \leq \mu_{1}$

$\sum_{u=1}^{U} c g_{u}^{A} t p_{u} \leq \mu_{2}$

$c g_{i}^{A}, c g_{u}^{A} \geq 0$

Where, $c g_{i}^{A}, c g_{u}^{A}$ stands for the instruction about the state of carrier among the $i^{\text {th }}$ and $u^{\text {th }} \mathrm{RRH}$ and the UE of the HPN. It is considered that the allotted frequency is common with UEs of RRHs. $\mu_{1}$ and $\mu_{2}$ is the granted ultimate interference from RRHs to UEs of HPN. Through (11), the optimal transmission power based on interference between RRH \& HPN among the UE can be inferred. By Integrating carrier gain and transmission power based on $i^{\text {th }}$ RRH cell and $\mathrm{u}^{\text {th }}$ HPN cell, granted ultimate interference of $\mu_{1}$ and $\mu_{2}$ shall be obtained through (12) \& (13).

Owing to its non-convexity characteristics, this issue is complicated to figure out. Hence it is required to be reconstructed to a different model in (14).

Let $\omega_{1}=\{1,1, \ldots \ldots 1\}_{N \times 1}^{T}$ and

$\omega_{2}=\frac{T P_{\max }}{\mu_{1}}+\frac{T P_{\max }}{\mu_{2}}\left\{c g_{1}^{A_{i}}+c g_{1}^{A_{u}}, c g_{2}^{A_{i}}+c g_{2}^{A_{u}}, \ldots . . c g_{i}^{A_{i}}+c g_{u}^{A_{u}}\right\}_{N \mathrm{x} 1}$ 
The constraints are

$\omega_{i, u}^{T} t p \leq T P_{\max }, \quad$ when $i, u=1,2$

Let $\vartheta_{i, u}^{z} \leq \log \left(1+\operatorname{SIN} R_{i, u}\right) \quad$ and $\quad \vartheta_{i, u}=\left(\vartheta^{1}, \vartheta^{2}, \ldots . . \vartheta^{i}\right)^{T}+\left(\vartheta^{1}, \vartheta^{2}, \ldots . \vartheta^{u}\right)^{T}$

Then the objective function becomes

$$
\begin{aligned}
& \sum_{i, u=1}^{I, U}\left(w t_{i}+w t_{u}\right) \vartheta_{i, u}^{z} \\
& e^{\vartheta_{i, u}^{z}}=1+\left[\frac{t p_{i}}{t p_{i} W M+a_{i}}+\frac{t p_{u}}{t p_{u} W F+a_{u}}\right]
\end{aligned}
$$

In matrix form,

$\left(\operatorname{diag}\left(e^{\vartheta_{i, u}^{z}}\right)-\mathrm{I}\right)\left[\left(t p_{i} W M+a_{i}\right)+\left(t p_{u} W F+a_{u}\right)\right]=t p_{i}\left(t p_{u} W F+a_{u}\right)+t p_{u}\left(t p_{i} W M+a_{i}\right)$

Diagonal matrix diag( ) with set of vector elements ai along with the transmission power of UE in (17) and the diag ( ) is simplified in (17).

Let $C=\left(t p_{i} W M+a_{i}\right)+\left(t p_{u} W F+a_{u}\right)$

And

Let $m=t p_{i}\left(t p_{u} W F+a_{u}\right)+t p_{u}\left(t p_{i} W M+a_{i}\right)$

It can be easily derived that

$\left(\operatorname{diag}\left(e^{\vartheta_{i, u}^{Z}}\right) c\right)=c+m$

Objective function ' $e$ ' and ' $\mathrm{j}$ ' is calculated based on the addition of transmission power of $\mathrm{u}^{\text {th }}$ and $\mathrm{i}^{\text {th }}$ RRH and HPN cell among UE represented in (16) and (18).

The above equation is to multiplied by

$J+\left(\frac{1}{T P_{\max }}\right) a w_{i, u}^{T}$

Where

$J=\frac{t p_{i}}{T p_{i} W M+a_{i}}+\frac{t p_{u}}{T p_{u} W F+a_{u}}$

hence the equation becomes:

$$
\begin{aligned}
& \left(J+\left(\frac{1}{T P_{\max }}\right) a w_{i, u}^{T}\right) \operatorname{diag}\left(e^{\vartheta_{i, u}^{Z}}\right) c=\left(J+\left(\frac{1}{T P_{\max }}\right) a w_{i, u}^{T}\right) c+\left(J+\left(\frac{1}{T P_{\max }}\right) a w_{i, u}^{T}\right) m \\
& \left(J+\left(\frac{1}{T P_{\max }}\right) a w_{i, u}^{T}\right) \operatorname{diag}\left(e^{\vartheta_{i, u}^{Z}}\right) c \leq\left(J+\left(\frac{1}{T P_{\max }}\right) a w_{i, u}^{T}\right) c+c
\end{aligned}
$$

Based on the integration of ' $\mathrm{J}$ ' objective function along with the diagonal matrix of $\mathrm{u}^{\text {th }}$ and $\mathrm{i}^{\text {th }} \mathrm{RRH}$ and HPN among UE as in (19). 


$$
\left(J+\left(\frac{1}{T P_{\max }}\right) a w_{i, u}^{T}\right) \operatorname{diag}\left(e^{\vartheta_{i, u}^{Z}}\right) c=c\left(1+J+\left(\frac{1}{T P_{\max }}\right) a w_{i, u}^{T}\right)
$$

Let $Q_{1}=J+\left(\frac{1}{T P_{\max }}\right) a w_{i, u}^{T}$

' $\mathrm{e}$ ' is denoted as the covariance of two difference concave diagonal matrix Q1 \& Q2 as represented in Eqn. 21.

Based on (19), again the covariance ' $e$ ' gets optimized based on $\mathrm{i}^{\text {th }}$ and $\mathrm{u}^{\text {th }} \mathrm{RRH}$ and HPN cell among UE as shown in (20). to calculate the covariance of two difference concave diagonal matrix Q1 \& Q2 as represented in (21) \& (22).

Now (16) is transformed to:

$Q_{1} \operatorname{diag}\left(e^{\vartheta_{i, u}^{Z}}\right) c \leq c\left(1+Q_{1}\right)$

Similarly

$Q_{2} \operatorname{diag}\left(e^{\vartheta_{i, u}^{z}}\right) c \leq c\left(1+Q_{2}\right)$

Then the problem is transformed in (23).

$\max \sum_{i, u=1}^{I, U}\left(w t_{i}+w t_{u}\right) e^{\vartheta_{i, u}^{Z}}$

Such that

$Q_{1} \operatorname{diag}\left(e^{\vartheta_{i, u}^{Z}}\right) c \leq c\left(1+Q_{1}\right)$

$Q_{2} \operatorname{diag}\left(e^{\vartheta_{i, u}^{Z}}\right) c \leq c\left(1+Q_{2}\right)$

Objective function formulation based on concave diagonal matrix optimization helps to optimize the interference and non-interference coordination with respect to resource allocation in C-RAN network.

Based on the above two cases namely, interference and non-interference coordination for either uplink/downlink helps to optimize the resource allocation and deployment of heterogeneous networks such as picocells and small cells, etc., with the integration of machine learning and C-RAN networks. Primary objective of the Co-ordinated UE system is to provide resource allocation to RRH cells effectively which leads to the QoS improvement to all the users in the network.

\section{V.Performance Analysis}

In the performance analysis, the Co-ordinated UE system is compared with three other existing systems namely HCRAN, EE-HCRAN and, Centralized resource allocation over the problem of energy efficiency. Simulation configuration for the performance analysis is represented in table. 1. 
Table. 1 Simulation Configuration

\begin{tabular}{|l|c|}
\hline \multicolumn{1}{|c|}{ Parameters } & Value \\
\hline Modulation Type & OFDMA_QPSK_1_2 \\
\hline Transmission Range & $500 \mathrm{~m}$ \\
\hline Packet Size & $1000 \mathrm{bits}$ \\
\hline User distribution & Uniform \\
\hline Total bandwidth & $20 \mathrm{MHz}$ \\
\hline Number of RRH & 100 \\
\hline RRH maximum transmission power & $25 \mathrm{dBm}$ \\
\hline Antenna gain for RRH & $6 \mathrm{~dB}$ \\
\hline Number of Resource Blocks & 1000 \\
\hline Traffic Demand & $6.4 \mathrm{Mbps} \sim 200 \mathrm{Mbps}$ \\
\hline
\end{tabular}

In the performance analysis, our Co-ordinated UE is compared with three other existing algorithms namely HCRAN, EE-HCRAN, centralized resource allocation. In this analysis, we have carried out the problem of energy efficiency.

\section{A. Analysis of Energy and spectral Efficiency:}

We have evaluated the performance of the Co-ordinated UE system with the existing system such as Centralized resource allocation [39] and EE-HCRAN to provide guaranteed QoS concerning energy and spectral efficiency. In our Co-ordinated UE system, we have included multi-tier H-CRAN architecture with the integration of machine learning which helps to improve the QoS. In the fig. 5, we have notified that our Co-ordinated UE system performs more after 400 iterations, i.e. time step in terms of energy efficiency which helps to achieve high data rate. The energy efficiency is calculated with respect to SINR threshold of data rate _ with T Pmax $=25 \mathrm{dBm}$. In the fig. 6 , we have analyzed that the Co-ordinated UE system is not sufficient compared to the existing systems until the SINR threshold is small as there is no more interference. When the limit gets more value, the proposed system gets it effectiveness to achieve data rate in terms of energy efficiency. In fig. 7, we have calculated the spectral efficiency with the Co-ordinated UE system by accessing the UE with $\mu_{1}$ and $\mu_{2}$ to achieve high spectral efficiency. Based on the spectral efficiency, the convergence time is achieved in the analysis. 


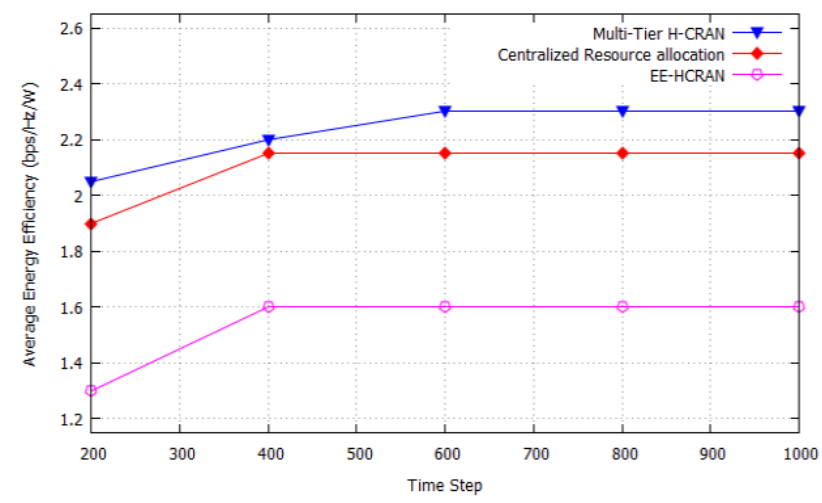

Fig. 5. Energy efficiency with different iterations

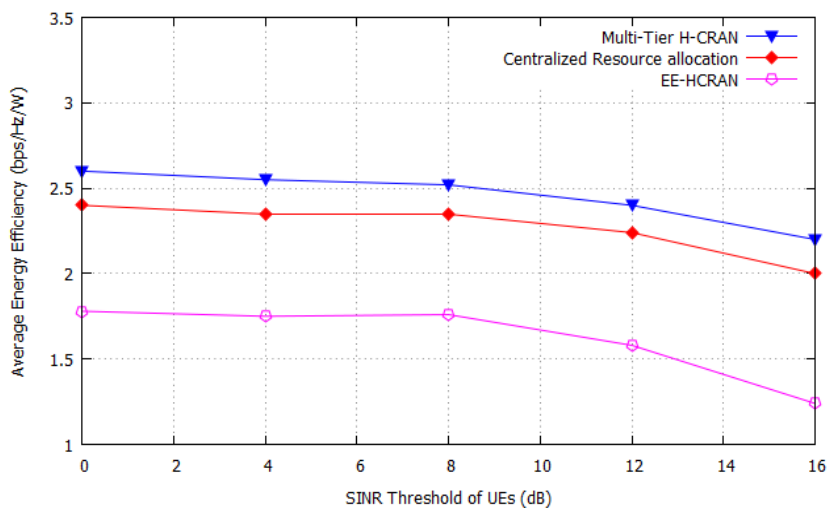

Fig. 6. Energy efficiency with SINR threshold

As inferred from the fig. 8, we have greater improvement in the power saving with the proposed multitier H-CRAN compared to the existing algorithms.

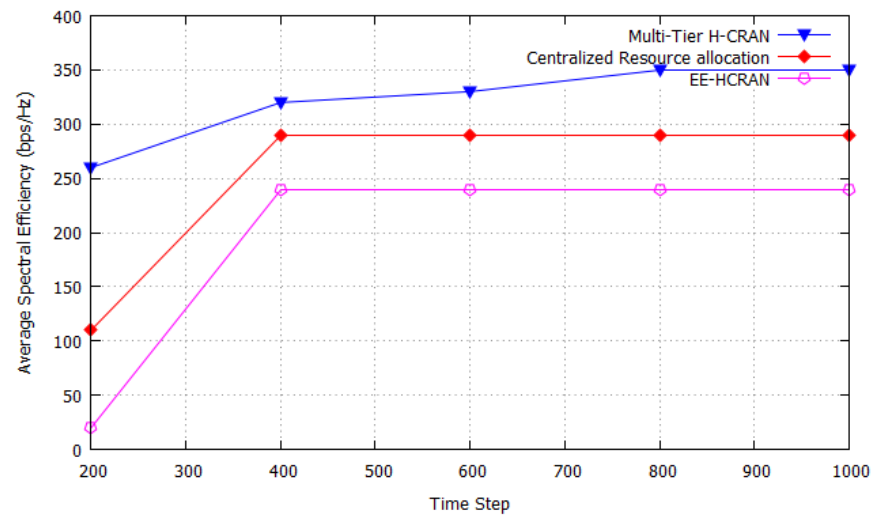

Fig. 7. Spectral efficiency with different iteration 


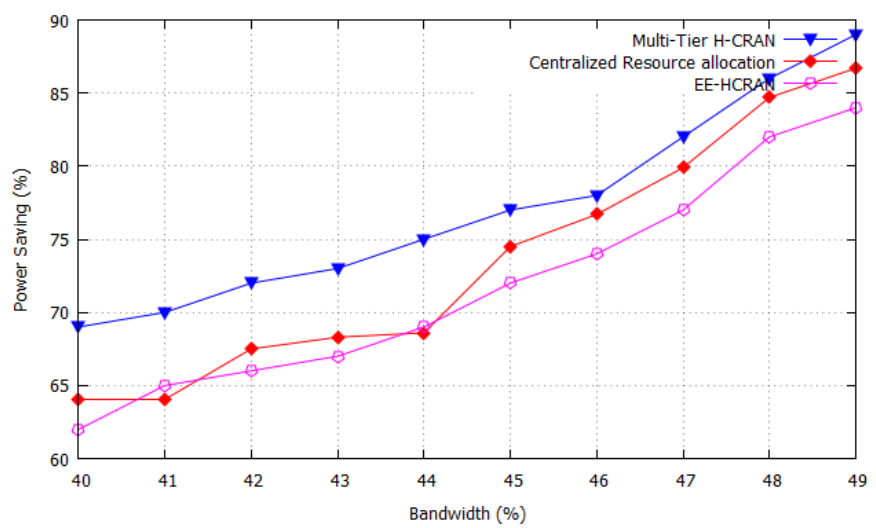

Fig. 8. Bandwidth Vs Power Saving

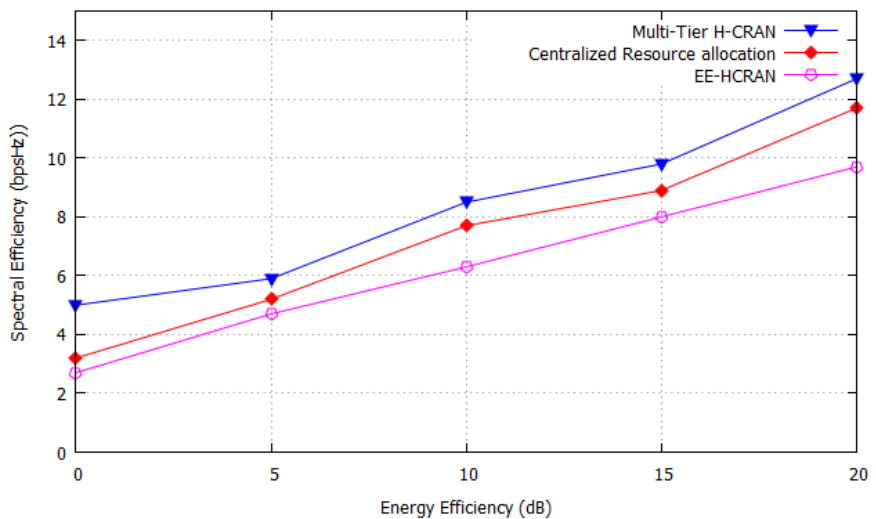

Fig. 9. Energy Efficiency Vs Spectral Efficiency

In the fig. 8, we have identified that the power saving is drastically increased by considering the variation in the bandwidth but the existing algorithms such as centralized Resource allocation and EE-HCRAN has more fluctuation in terms of power saving. As inferred from the fig. 8, we have more significant improvement in the power saving with the proposed multi-tier H-CRAN compared to the existing algorithms. In the fig. 9, we have analysis the energy efficiency with the spectral efficiency and we inferred that the spectral efficiency gets improved for our proposed multi-tier H-CRAN compared to the existing algorithms such as, centralized Resource allocation and EE-HCRAN.

B. Analysis of Data Rate with the variation sets in the resource block:

Fig. 10, 11 and 12 evaluated the data rate with respect to Commutative Distribution Function $(\mathrm{CDF})$ by accessing the UE of _ based on the resource block where the resource block $=1000$. By varying the resource block sets, we have analysed that the Co-ordinated UE system gets its efficiency based on the above threshold of 2, 0.5 and 4 (Mbps) compared to the existing systems. 


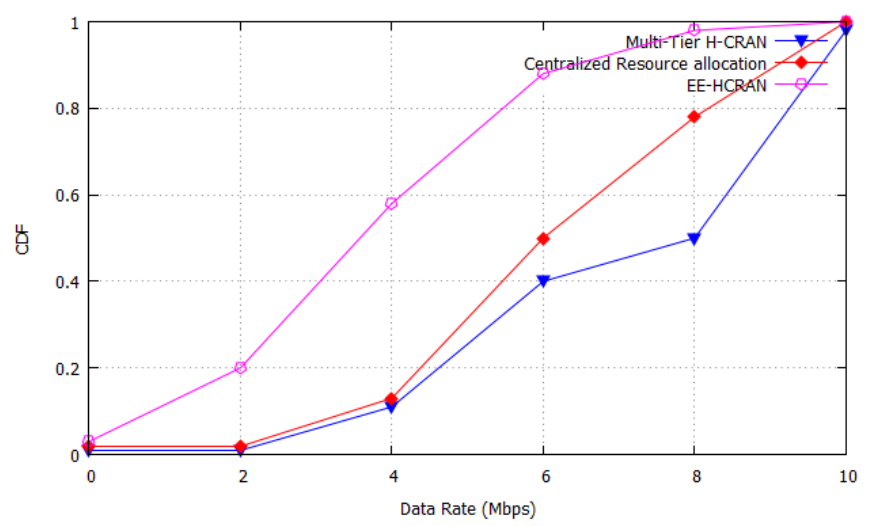

Fig. 10. CDF data rate by accessing $\mathrm{UE} \mathrm{RB} 1$

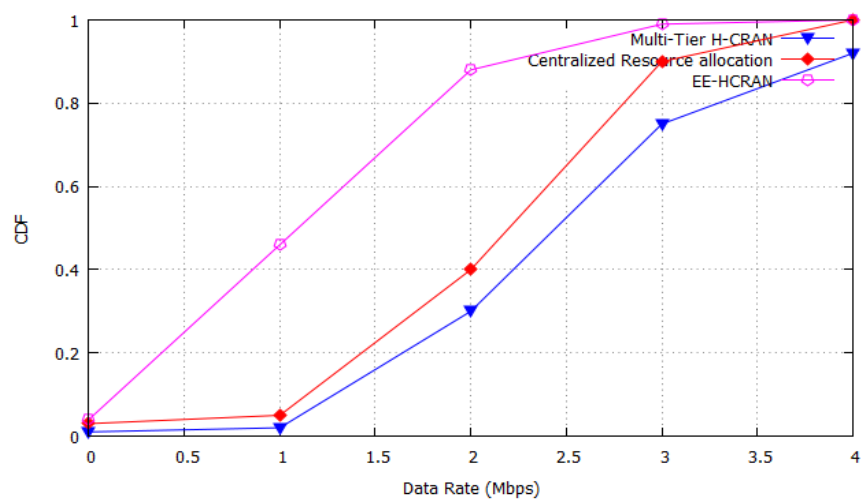

Fig. 11. CDF data rate by accessing $\mathrm{UE} \mathrm{RB}_{2}$

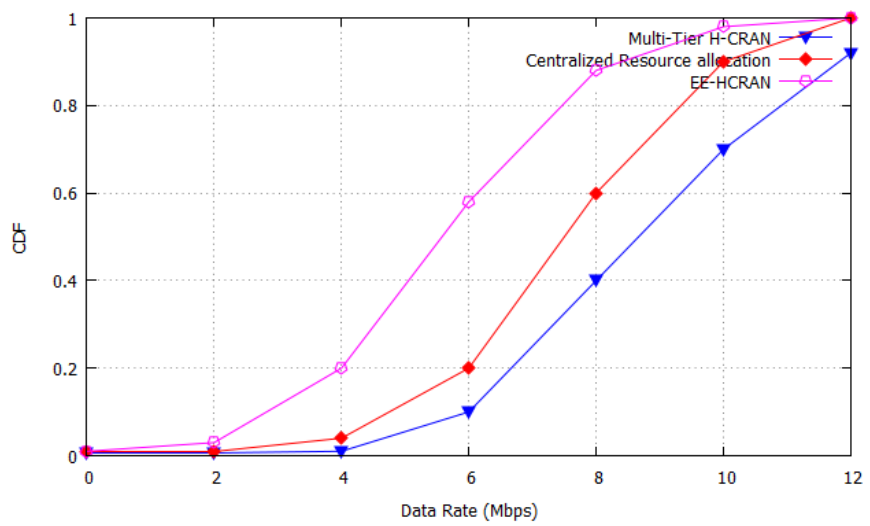

Fig. 12. CDF Data rate by accessing the $\mathrm{UE} \mathrm{RB}_{3}$

\section{Analysis of throughput with respect to network constraints:}

In fig. 13, aggregated throughput is calculated based on the variation in the number of users from 0.0084 to $0.0121 / \mathrm{m}^{2}$. The throughput gets increased as the number of users also gets increased for the Co-ordinated UE system compared to the existing systems such as centralized resource allocation and EE-HCRAN. 


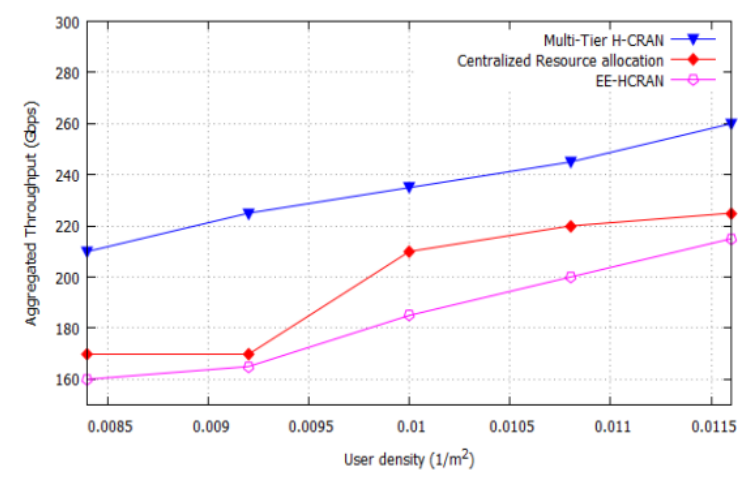

Fig. 13. Throughput Vs User density

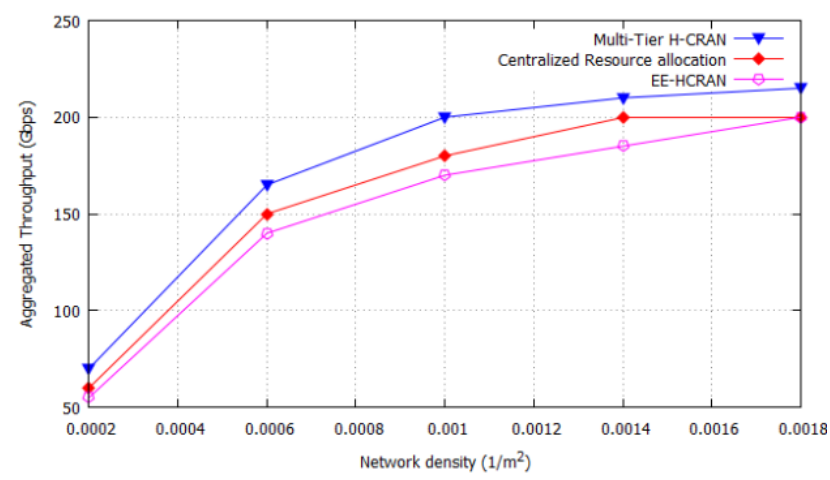

Fig. 14. Throughput Vs Network density

Throughput is calculated based on the variation in the number of network from 0.0002 to $0.0181 / \mathrm{m}^{2}$. As the throughput gets equalize for the existing systems when the network density gets increased. The Co-ordinated UE system gets improved throughput based on the increase in the number of network density in fig. 14.

In fig. 15, the average throughput is performed by setting up the equal transmit power for all RRH. For the simulation, the maximal power of transmission is fixed at 0.5 and $1.0 \mathrm{~W}$. By varying the transmit power, Co-ordinated UE system gets increased throughput by increasing the transmit power. The throughput gets loss in the performance due to the high interference among RRH's in the network. The transmit power gets increases, throughput also gets increased and it is illustrated in fig. 16. Here, interference $\mu$ from RRH increases, the throughput also gets increased by increasing the allocated transmit power.

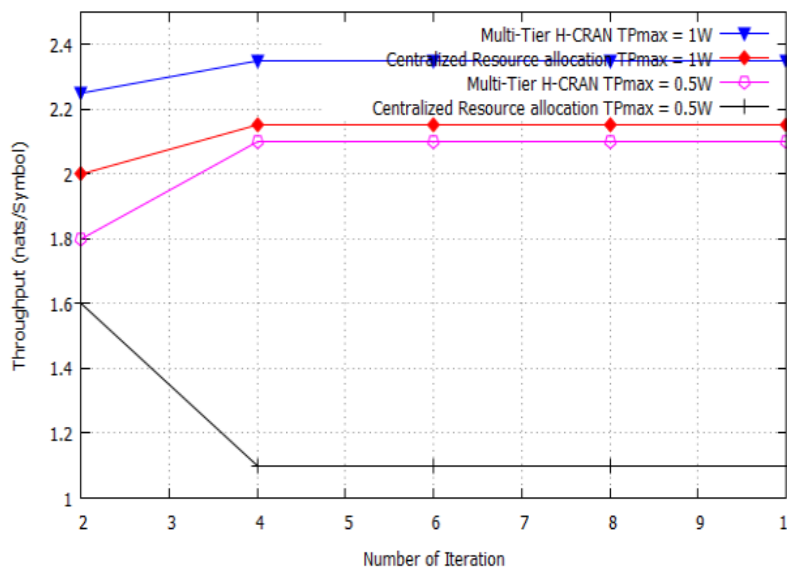

Fig. 15. Throughput Convergence

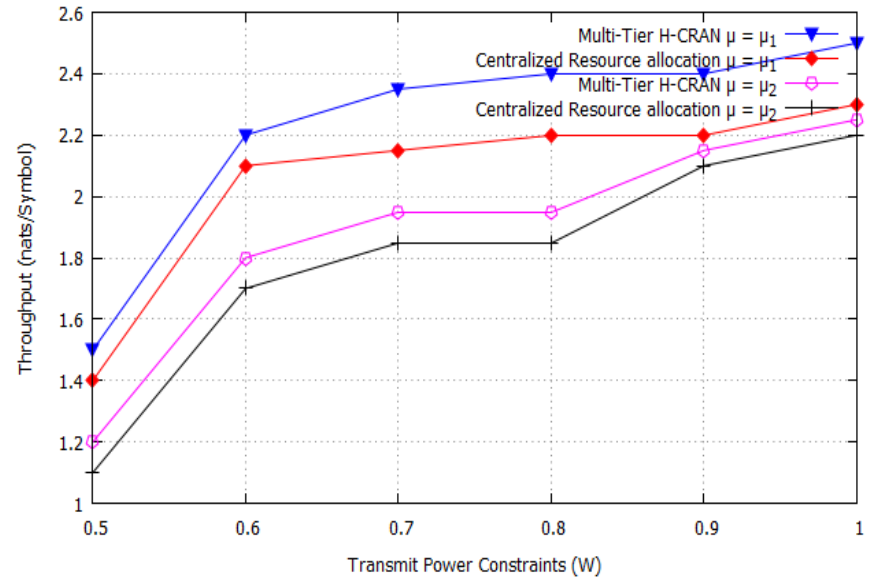

Fig. 16. Throughput Performance with different $\mathrm{TP}_{\max }$

In fig. 17, the color proportion is calculated for the Co-ordinated UE system based on the number of oscillation. The more colors gets less problem at the boundary of radio in each oscillation. If each cell is assigned with the same color will decrease the convergence based on the number of iteration. RAN can also be analyzed based on security issues such as confidentiality and authentication based on UE prediction [40]. 


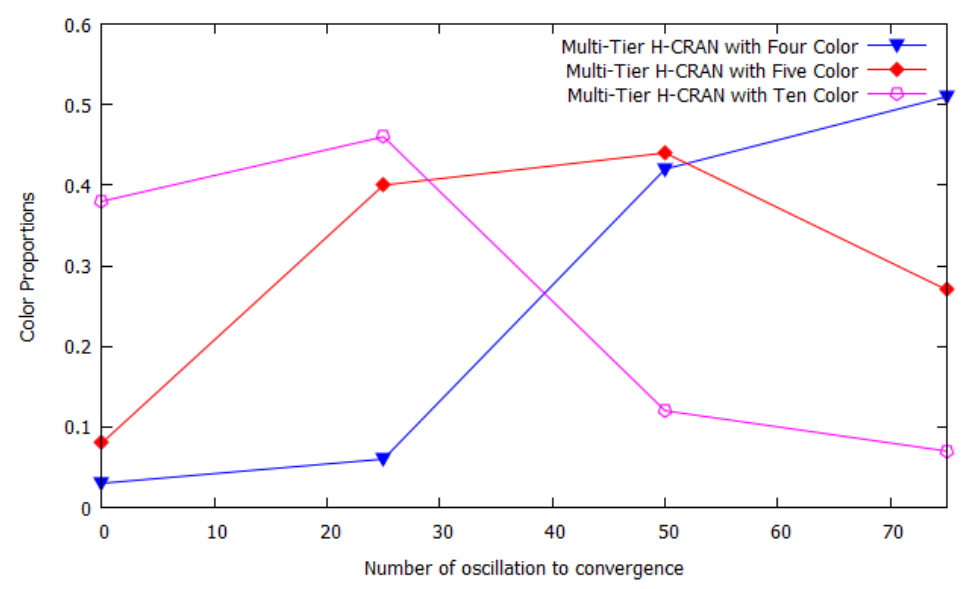

Fig. 17. Color proportion Vs Number of oscillation convergence

\section{Conclusion}

As user expectations are increasing every day, networks are also evolving accordingly. The future network should provide seamless service with minimal latency by high resource utilization. In this article, we considered various problems that arise when different spectrum bands operate under one umbrella and we have effectively managed to provide an interoperability architecture for the future evolving 5G network. In this work, we proposed a Multi-tier H-CRAN architecture, that incorporates heterogeneous networks to operate under a single controller by predicting the user expectation and selecting the desired network based on the user profile. The machine learning paradigm is used to learn the user profiles and network conditions under different payload and we simulated the real time traffic through the network simulator. The proposed mechanism have been analysed under several conditions through simulation and we found that adopting a machine learning paradigm to the C-RAN paves the way for providing intelligence in the network selection. Also, our mathematical analysis reveals that adaptability of the proposed architecture is smooth and highly scalable. The future work of this article is planned to be extended for strengthening the bits of intelligence for the decision making in network selection and to provide optimal resource allocation.

\section{REFERENCES}

[1] Blanco B, Fajardo JO, Giannoulakis I, Kafetzakis E, Peng S, Pérez-Romero J, et al. Technology pillars in the architecture of future 5G mobile networks NFV MEC and SDN. Computer Standards and Interfaces Elsevier Publications 2017;54(2):216-228.

[2] Taleb T, Samdanis K,MadaB, Flinck H, Dutta S, SabellaD. OnMulti-Access Edge Computing: ASurvey of the Emerging5G Network Edge Cloud Architecture and Orchestration. IEEE Communications Surveys and Tutorials 2017;19(3):1657-1681.

[3] Ramanathan R, Jayakumar M. A support vector regression approach to detection in large-MIMO systems. Telecommunication Systems, Springer link 2017;64(4):709-717.

[4] Sarayu S, Radhakrishnan J, Kirthiga S. Superimposed Pilot Based Channel Estimation forMIMOSystems. In: Proceedings of Artificial Intelligence and Evolutionary Computations in Engineering Systems; 2017.p. $115-126$.

[5] Tzanakakia A, Anastasopoulos M, Berberana I, Syrivelis D, Flegkas P, Korakis T, et al. Wireless-Optical Network Convergence: Enabling the 5G Architecture to Support Operational and End-User Services. IEEE Communications Magazine 2017;55(10):184-192. 
[6] Foukas X, Patounas G, Elmokashfi A, Marina MK. Network Slicing in 5G: Survey and Challenges. IEEE Communications Magazine 2017;55(3):94-100.

[7] Jiang C, Zhang H, Ren Y, Han Z, ChenKC, Hanzo L. Machine Learning Paradigms forNext-Generation Wireless Networks. IEEE Wireless Communications 2017;24(2):98-105.

[8] Arul R, Raja G, Bashir AK, Chaudry J, Ali A. A Console GRID Leveraged Authentication and Key Agreement Mechanism for LTE/SAE. IEEE Transactions on Industrial Informatics 2018;14(6):26772689.

[9] Sundaresan K, Arslan MY, Singh S, Rangarajan S, Krishnamurthy SV. FluidNet: A Flexible Cloud-Based Radio Access Network for Small Cells. IEEE/ACMTransactions on Networking 2016;24(2):915-928.

[10] Wang Z, Li H,Wang H, Ci S. Probability weighted based spectral resources allocation algorithm in Hetnet under Cloud- RAN architecture. In: Proceedings of IEEE/CIC International Conference on Communications in China - Workshops (CIC/ICCC); 2013; 88-92.

[11] Pérez-Romero J, Sánchez-González J, Sallent O, Agustí R. On Learning and Exploiting Time Domain Traffic Patterns in Cellular Radio Access Networks. Machine Learning and Data Mining in Pattern Recognition; Springer LNCS 2016; 9729:501--515.

[12] Bastug E, Bennis M, Debbah M. Living on the edge: The role of proactive caching in $5 \mathrm{G}$ wireless networks. IEEE Communications Magazine 2014;52(8):82-89.

[13] Marsch P, Silva ID, BulakciO, Tesanovic M, Ayoubi SEE, Rosowski T, et al. 5G Radio Access Network Architecture: Design Guidelines and Key Considerations. IEEE Communications Magazine 2016;54(11):24-32.

[14] Yang C, Chen Z, Xia B,Wang J. When ICN meetsC-RAN for HetNets: an SDN approach. IEEE Communications Magazine 2015;53(11):118-125.

[15] Hattachi RE, Erfanian J. 5G White Paper-NGMN5G Initiative. NGMNAlliance 2015.

[16] Chabbouh O, Rejeb SB, Choukair Z, Agoulmine N. A novel cloud RAN architecture for 5G HetNets and QoS evaluation. In: Proceedings of International Symposium on Networks, Computers and Communications (ISNCC); 2016.

[17] Bashir AK, Lim SJ, Hussain CS, Park MS. Energy Efficient In-network RFID Data Filtering Scheme in Wireless Sensor Networks. Sensor MDPI 2011;11(7):7004-7021.

[18] Gerasimenko M, Moltchanov D, Florea R, Andreev S, Koucheryavy Y, Himayat N, et al. Cooperative Radio Resource Management in Heterogeneous Cloud Radio Access Networks. IEEE Access 2015;3:397406.

[19] Ran C,Wang S,Wang C. Balancing backhaul load in heterogeneous cloud radio access networks. IEEE Wireless Communications 2015;22(3):42-48.

[20] Liu C, Sundaresan K, Jiang M, Rangarajan S, Chang GK. The case for re-configurable backhaul in cloudRAN based small cell networks. In: Proceedings of IEEE INFOCOM; 2013.

[21] Chen L, Jin H, Li H, Seo JB, Guo Q, Leung V. An Energy Efficient Implementation of C-RAN in HetNet. In: Proceedings of Vehicular Technology Conference (VTC Fall); 2014.

[22] Palicot J, Zhang H, Moy C. On the road towards green radio. URSI Radio Science Bulletin 2013;2013(347):40-56.

[23] Wang X, Li X, Leung VCM. Artificial Intelligence-Based Techniques for Emerging Heterogeneous Network: State of the Arts, Opportunities and Challenges. IEEE Access 2015;3:1379-1391.

[24] Peng M, Liu Y,Wei D,WangW, Chen HH. Hierarchical cooperative relay based heterogeneous networks. IEEE Wireless Communications 2011;18(3):48-56.

[25] Jiang C, Zhang H, Ren Y, Han Z, ChenKC, Hanzo L. Machine Learning Paradigms forNext-Generation Wireless Networks. IEEE Wireless Communications 2017;24(2):28-105.

[26] Foukas X, Patounas G, Elmokashfi A, Marina MK. Network Slicing in 5G: Survey and Challenges. IEEE Communication Magazine 2017;55(5):94-100. 
[27] Jayaraman R, Raja G, Bashir AK, Hussain CS, Ali Hassan MAA. Interference Mitigation Based on Radio Aware Channel Assignment for Wireless Mesh Networks. Wireless Personnal Communication Springer publications 2018;

[28] Temesgene DA, Nunez-Martinez J, Dini P. Softwarization and Optimization for Sustainable Future Mobile Networks: A Survey. IEEE Access 2017;5:25421-25436.

[29] AlQerm I, Shihada B. Enhanced machine learning scheme for energy efficient resource allocation in 5G heterogeneous cloud radio access networks. In: Proceedings of IEEE 28th Annual International Symposium onPersonal, Indoor, and Mobile Radio Communications (PIMRC); 2017.

[30] Lien SY, Hung SC, Deng DJ,Wang YJ. Efficient Ultra-Reliable and Low Latency Communications and Massive Machine- Type Communications in 5G New Radio. In: Proceedings IEEE Global Communications Conference of GLOBECOM; 2017.

[31] Tsiropoulos GI, Yadav A, Zeng M, DobreOA. Cooperation in 5G HetNets: Advanced Spectrum Access and D2D Assisted Communications. IEEE Wireless Communications 2017;24(5):110-117.

[32] Guizani Z, Hamdi N. CRAN, H-CRAN, and F-RAN for 5G systems: Key capabilities and recent advances. International Journal of Network Management Wiley online Library 2017;27(5).

[33] Huang PH, Kao H, LiaoW. Hierarchical Cooperation in Heterogeneous Cloud Radio Access Networks. In: Proceedings International Conference on Communications (ICC); 2016

[34] Boulos K, Helou ME, Ibrahim M, Khawamy K, Sawaya H, Martin S. Interference-Aware Clustering in Cloud Radio Access Networks. In: Proceedings IEEE 6th conference on Cloud Networking (CloudNet); 2017.

[35] Jayaraman R, Raja G, Ghosal D, Arul R, A SK. A Compatibility Vector Technique for Cooperative Scheduling and Channel Assignment Algorithm in Broadband Wireless Networks. ACM- Springer Journal of Mobile Networks and Applications (MONETS) 2017;22(4):730-742.

[36] Ahmad SA, DatlaD. Distributed Power Allocations in Heterogeneous Networks With Dual Connectivity Using Backhaul State Information. ACM- Springer - Journal of Mobile Networks and Applications (MONETS) 2017;22(4):730-742.

[37] Jiang P, Bigham J, Khan MA. Distributed Algorithm for Real Time Cooperative Synthesis of Wireless Cell Coverage Patterns. IEEE Communications Letters 2008;12(9):702-704.

[38] Du L, Biaham J, Cuthbert L. A bubble oscillation algorithm for distributed geographic load balancing in mobile networks. In: Proceedings of Twenty-third AnnualJoint Conference of the IEEE Computer and Communications Societies INFOCOM; 2004.

[39] Peng M, Zhang K, J Jiang JW, Wang W. Energy efficient resource assignment and power allocation in heterogeneous cloud radio access networks. IEEE Transactions on Vehicular Technology 2015;64(11):5275-5287.

[40] Arul R, Raja G, Kottilingam Kottursamy SP, Venkatraman S. User Path Prediction Based Key Caching and Authentication Mechanism for Broadband Wireless Networks. Springer - Journal on Wireless Personal Communications 2017;94(4):2645-2664.

[41] I . F. Siddiqui, S.U. J. Lee, A. Abbas, and A.K. Bashir. Optimizing Lifespan and Energy Consumption by Smart Meters in Green-Cloud-Based Smart Grids. IEEE Access, vol. 5, pp. 20394-20945, 2017

[42] Shakil M, A.F.Y. Mohammed, Arul R, A.K. Bashir, and J.K. Choi. A Novel Dynamic Framework to detect DDoS in SDN using Meta Heuristic Clustering. Wiley - Transactions on Emerging Telecommunications Technologies 2019; Accepted in Production. 